\title{
Nonlinear Entanglement and its Application to Generating Cat States
}

\author{
Y. Shen, ${ }^{1,2,3}$ S. M. Assad, ${ }^{2}$ N. B. Grosse, ${ }^{2}$ X. Y. Li, ${ }^{1}$ M. D. Reid, ${ }^{4}$ and P. K. Lam ${ }^{1,2, *}$ \\ ${ }^{1}$ College of Precision Instrument and Optoelectronics Engineering, \\ Key Laboratory of Optoelectronics Information Technology of Ministry of Education, \\ Tianjin University, Tianjin 300072, China \\ ${ }^{2}$ Centre for Quantum Computation and Communication Technology, Research School of Physics and Engineering, \\ Australian National University, Canberra, Australian Capital Territory 0200, Australia \\ ${ }^{3}$ Department of Physics, The National University of Defense Technology, Changsha 410073, China \\ ${ }^{4}$ Centre for Atom Optics and Ultrafast Spectroscopy, Swinburne University of Technology, Melbourne, Victoria 3122, Australia
}

(Received 21 May 2014; revised manuscript received 21 September 2014; published 10 March 2015)

\begin{abstract}
The Einstein-Podolsky-Rosen (EPR) paradox, which was formulated to argue for the incompleteness of quantum mechanics, has since metamorphosed into a resource for quantum information. The EPR entanglement describes the strength of linear correlations between two objects in terms of a pair of conjugate observables in relation to the Heisenberg uncertainty limit. We propose that entanglement can be extended to include nonlinear correlations. We examine two driven harmonic oscillators that are coupled via third-order nonlinearity can exhibit quadraticlike nonlinear entanglement which, after a projective measurement on one of the oscillators, collapses the other into a cat state of tunable size.
\end{abstract}

The argument developed in 1935 by Einstein, Podolsky, and Rosen (EPR) [1] illuminated a striking inconsistency: quantum mechanics, as it stood then and still stands today, is a theory incompatible with the premise of local realism. Although never doubting the validity of the theory in describing the outcomes of measurements, EPR concluded that quantum mechanics was conceivably an incomplete description of reality. At the heart of the EPR paradox is the ideal case of two particles that are spatially separated, yet exhibit both perfect linear correlation in momentum and anticorrelation in position when measurements of each are compared. This set of correlations for a conjugate pair of observables, termed by Schrödinger as the phenomenon of steering [2], requires the wave function describing both particles to be inseparable, in which the two particles are said to be entangled. Hence, two particles that invoke the EPR paradox, i.e., can be steered, are defined as being EPR entangled [3-5]. The requirement of steerability is strictly stronger than that of entanglement (wave function inseparability), and strictly weaker than that of the Bell nonlocality [6-9].

The entangled states of massive particles that were introduced in the original EPR paper, possessing correlations in continuous variables (CVs), have so far escaped verification. However, it was discovered that pairs of particles of the electromagnetic field could be created via the process of optical parametric oscillation. The inseparable wave function of the resulting two-mode squeezed state [10] has since been used to demonstrate steerability, the EPR paradox [11-15], and a diverse command of quantum information protocols. Surprisingly, only linear correlations leading to EPR entanglement have been considered thus far. We propose that nonlinear correlations in $\mathrm{CV}$ observables can lead to a form of nonlinear entanglement that demonstrates steerability by satisfying an equivalent nonlinear EPR criterion.

In this Letter, we develop the concept of nonlinear EPR entanglement and propose a method for demonstrating it optically via a third-order nonlinear interaction. We reveal how a cat state of tunable size is produced by a simple projective measurement of the quadraticlike correlations that naturally comprise the nonlinearly entangled state.

Let us consider a three-photon down-conversion process, where a pump photon at frequency $\omega_{C}$ is down converted to a signal photon at frequency $\omega_{A}$ and two degenerate idler photons at frequency $\omega_{B}$, with the energy conservation relation $\omega_{C}=\omega_{A}+2 \omega_{B}$. This process is described by the Hamiltonian

$$
\hat{H}=\AA \hbar \kappa\left(\hat{a}^{\dagger} \hat{b}^{\dagger 2} \hat{c}-\hat{a} \hat{b}^{2} \hat{c}^{\dagger}\right),
$$

where $\kappa$ is the third-order coupling constant that describes the strength of the nonlinear interaction, $\hat{a}$ and $\hat{b}$ are the annihilation operators of the down-converted signal and idler modes, and $\hat{c}$ is the annihilation operator for the pump mode.

With this Hamiltonian we propagate the initial state of no photons in the signal and idler modes and a coherent state $\left|\alpha_{c}\right\rangle$ in the pump mode for a time $t$. Using the method introduced in Ref. [16], we can obtain the generated state numerically.

Because of the down-conversion process induced by the pump, the modes $A$ and $B$ evolve away from their initial vacuum states. This is evident in the joint probability distributions of the linear quadratures taken between modes $A$ and $B$, as shown in Fig. 1. These distributions, which 


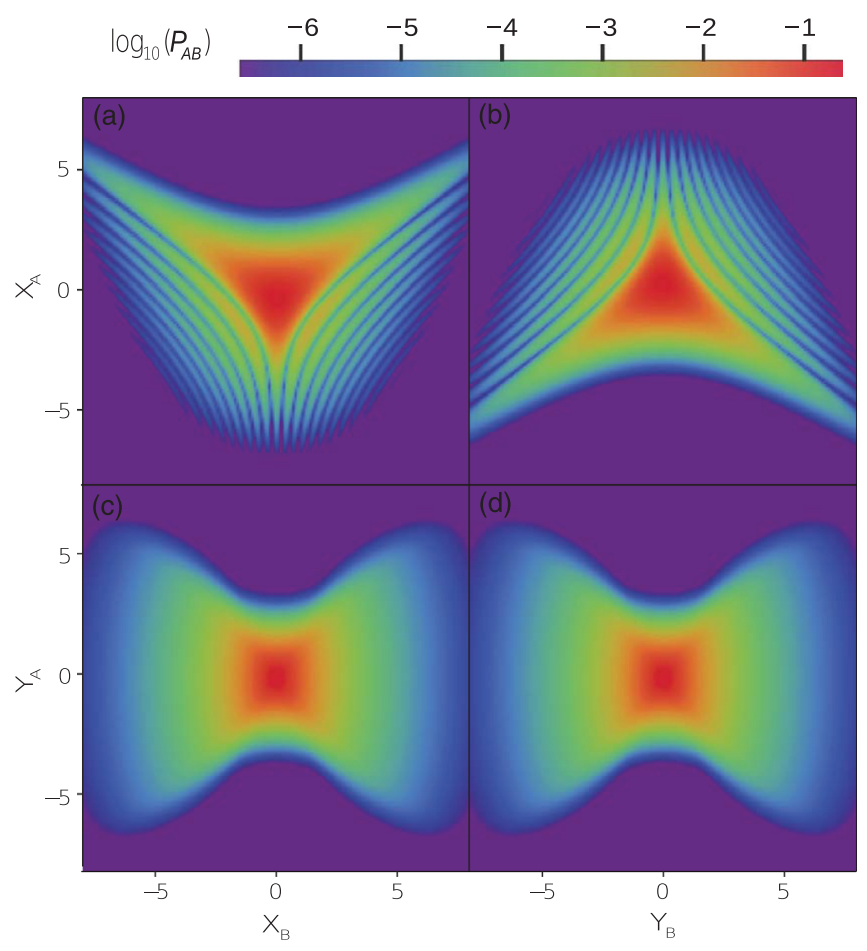

FIG. 1 (color). Linear quadrature joint probability distributions for (a) $X_{A}$ and $X_{B}$, (b) $X_{A}$ and $Y_{B}$, (c) $Y_{A}$ and $X_{B}$, (d) $Y_{A}$ and $Y_{B}$. The joint probability distributions between the quadrature amplitudes of modes $A$ and $B$ displays no linear correlation. Interaction strength $\alpha_{c} \kappa t=0.3$ and pump amplitude $\alpha_{c}=\sqrt{10}$.

initially were Gaussian and circularly symmetric, now show a great deal of complexity, and hint at the nonlinear correlation properties of the final two-mode state.

Being a key element for quantum information processing [17-21], the cat state has been experimentally realized using several approaches [22-25]. However, to date, the size of the state has not been readily tunable. In our proposal, the projective measurement is a continuous variable, and, hence, allows the size of the cat state to be tuned continuously. Figures 2(a)-2(d) shows the cross sections of the joint probability distributions for values of $X_{A}=1,2,3$, and 4 . Here we have taken the scaling $\hbar=1$. These give the probability distribution of $X_{B}$ given a particular outcome of $X_{A}$. For large-enough $X_{A}$, we observe two distinct peaks in $X_{B}$, which become more separated with larger $X_{A}$. This displays the coherent superposition phenomena where two contradictory outcomes are simultaneously possible. The interference fringes in the orthogonal mode $\hat{Y}_{B}$ tell us that the projected state cannot be a statistical mixture of two states.

The Wigner function of the projected mode $\hat{\rho}_{B}$ for the outcome $X_{A}=3$ is plotted in Fig. 3(a). We find that it bears a remarkable resemblance to the conventional optical cat state $\left|\alpha_{0}\right\rangle+\left|-\alpha_{0}\right\rangle$ [26]. The fidelity between the generated state and the cat state with $\alpha_{0}=2.9$ is 0.943 .

For completeness, we also plot the Wigner function of mode $A$ for an outcome on $X_{B}=3$ in Fig. 3(b). It shows an

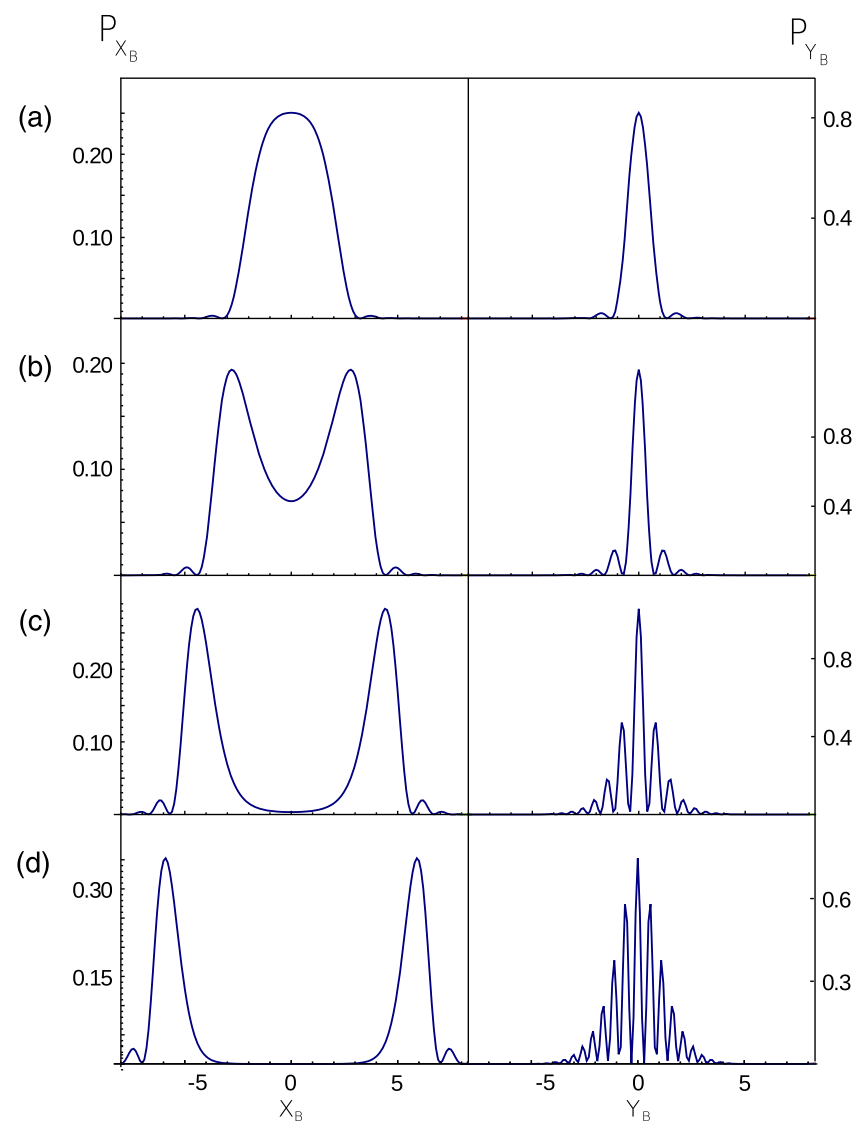

FIG. 2 (color online). Tuning a cat state. The probability distributions of the quadratures $X_{B}$ (left) and $Y_{B}$ (right) when postselecting with (a) $X_{A}=1$, (b) $X_{A}=2$, (c) $X_{A}=3$, and (d) $X_{A}=4$. The cat state amplitude increases with $X_{A}$. Interaction strength $\alpha_{c} \kappa t=0.3$ and pump amplitude $\alpha_{c}=\sqrt{10}$.

asymmetric parabolic distribution with a marked negativity and interference pattern near the center. In spite of the dissimilar appearance, both Wigner functions in Figs. 3(a) and 3(b) exhibit strong coherent superposition characteristics, as seen by the ripples in the quadrature projections.

Next, we shall show that the modes $A$ and $B$ are not only correlated, but also entangled. As an entanglement witness, we use the negativity $N(\hat{\rho})$ defined as [29]

$$
N(\hat{\rho})=\frac{\left\|\hat{\rho}^{T_{B}}\right\|-1}{2},
$$

where $\hat{\rho}^{T_{B}}$ is the partial transpose over mode $B$ and $\|A\|=\operatorname{Tr}\left(\sqrt{A^{\dagger} A}\right)$ is the trace norm. $N>0$ is the sufficient condition of entanglement.

Since the photons in modes $A$ and $B$ are generated simultaneously, we expect them to be entangled. For small interaction strength, the three-mode state is approximately $\left(\left|0_{A} 0_{B}\right\rangle+\left|1_{A} 2_{B}\right\rangle \alpha_{C} \kappa t\right)\left|\alpha_{c}\right\rangle$, which is entangled between modes $A$ and $B$. This is shown in the negativity plots in Fig. 4(a). In Fig. 4(b), we see that for short interaction strength $\left(\alpha_{c} \kappa t<0.4\right)$, the state $\hat{\rho}_{A B}$ maintains a purity 


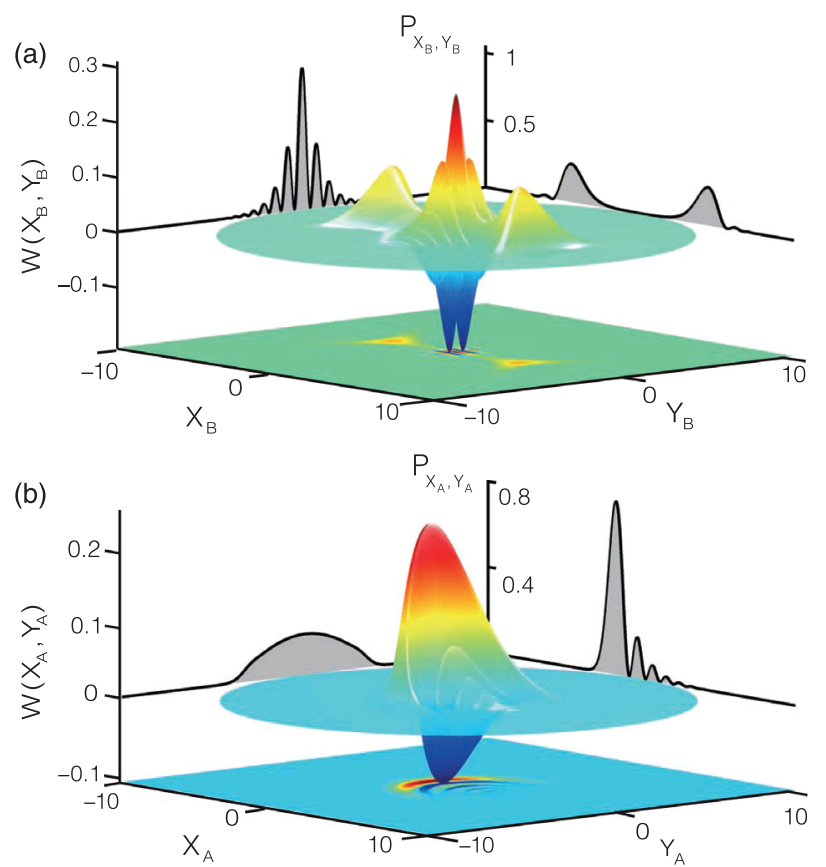

FIG. 3 (color). Wigner function of postselected state. (a) Wigner function of projected mode $B$ when $X_{A}=3$, displaying features of a cat state. (b) Wigner function of projected mode $A$ when $X_{B}=3$. The projections on the vertical planes show the probability density distribution of a quadrature measurement. The horizontal plane projection gives a contour plot of the Wigner function. Interaction strength $\alpha_{c} \kappa t=0.3$ and pump amplitude $\alpha_{c}=\sqrt{10}$

higher than 0.9. We also see in these figures that the entanglement strength and purity increase for a brighter pump and a correspondingly weaker coupling constant $\kappa$. This trend has its origins in the fact that a brighter pump has reduced depletion and, hence, approaches the parametric approximation [30,31].

Steerability is also exhibited in this two-mode state between the normal linear quadratures of mode $A$ and the so-called squared amplitudes [32] of mode $B$, which are defined as

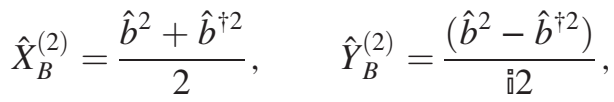

where $\hat{b}$ and $\hat{b}^{\dagger}$ are the annihilation and creation operators for mode $B$.

To quantify the steerability of mode $B$ by mode $A$, we shall use the $j$ th steerability index $R^{(j)}$ [33], which is defined as [26]

$$
R^{(j)}=\frac{2 \sqrt{\left\langle V\left(\hat{X}_{B}^{(j)} \mid X_{A}\right)\right\rangle\left\langle V\left(\hat{Y}_{B}^{(j)} \mid Y_{A}\right)\right\rangle}}{\left\langle\left[\hat{X}_{B}^{(j)}, \hat{Y}_{B}^{(j)}\right]\right\rangle},
$$

where $V\left(\hat{O}_{B} \mid O_{A}\right)$ is the variance of $\hat{O}_{B}$ when the measurement of $\hat{O}_{A}$ on mode $A$ gives the outcome $O_{A}$, and
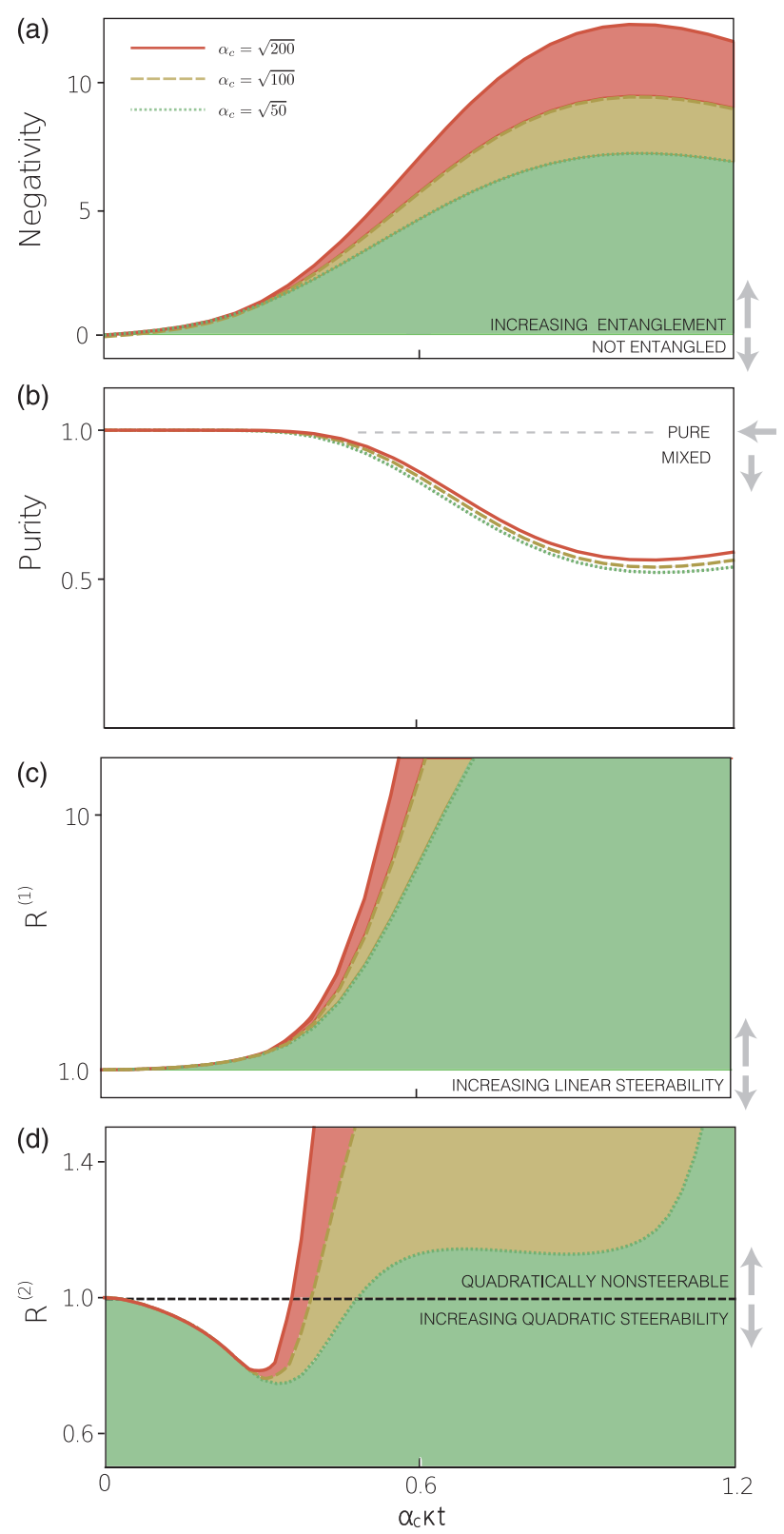

FIG. 4 (color). (a) Entanglement, (b) purity, (c) linear steerability, and (d) quadratic steerability of the generated state. (a) We observe a positive negativity for all interaction time indicating entanglement between modes $A$ and $B$. (b) The state $\rho_{A B}$ has a high purity when $\alpha_{c} \kappa t<0.3$. (c) The linear quadratures are not EPR steerable. (d) However, the quadratic quadratures displays EPR steering when $\alpha_{c} \kappa t<0.3$. A weak third-order coupling constant $\kappa$ can be compensated by using a stronger pump $\alpha_{c}$.

$\left\langle V\left(\hat{O}_{B} \mid O_{A}\right)\right\rangle$ is the average of $V\left(\hat{O}_{B} \mid O_{A}\right)$ over all the possible outcomes $O_{A}$. Here $\hat{X}^{(1)}, \hat{Y}^{(1)}$ are just the normal linear quadratures $\hat{X}, \hat{Y}$. The $j$ th quadrature mode $B$ is steerable by mode $A$ if $R^{(j)}<1$. When this inequality is satisfied, on average both the amplitude and phase $j$ th quadrature variances of $B$ can be "squeezed" by measuring the appropriate quadrature of $A$, giving an apparent violation of the Heisenberg uncertainty relation and, hence, 


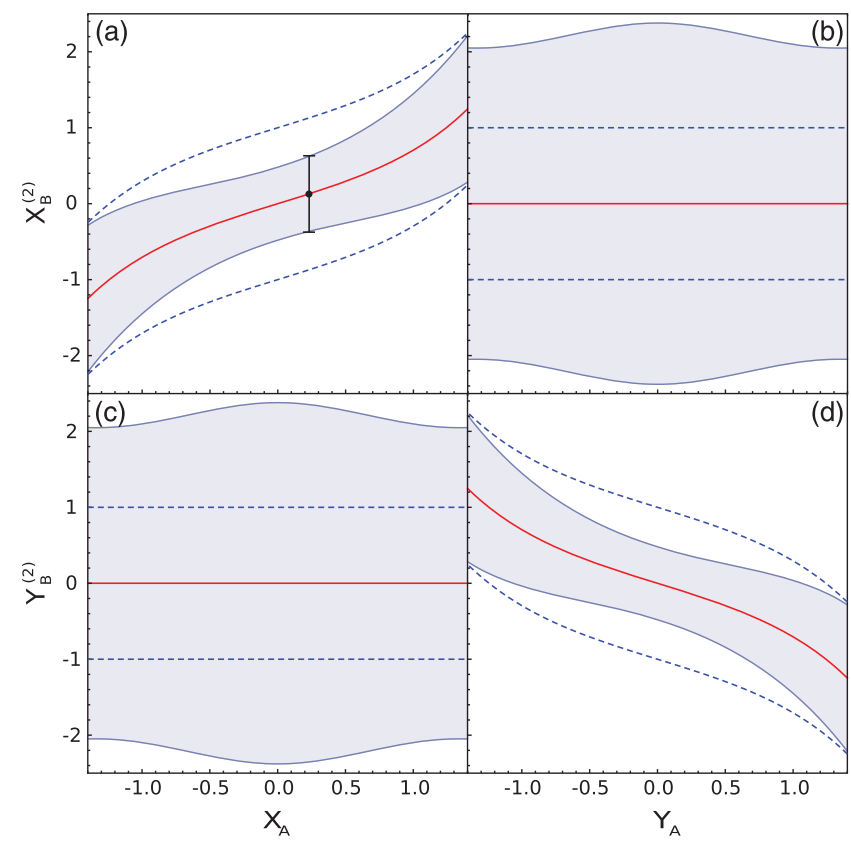

FIG. 5 (color). Quadratic quadrature conditional joint probability distributions between modes $A$ and $B$. Mean value of the quadratic quadrature of mode $B$ conditioned on a linear quadrature value of mode $A$ (red trace). The shaded region displays the normalized standard deviation of the distribution and the blue dashed trace gives the Heisenberg uncertainty limit. Interaction strength $\alpha_{c} \kappa t=0.3$ and pump amplitude $\alpha_{c}=\sqrt{10}$.

an EPR paradox [1,33]. For $j=1$, this is similar to the well-known Duans inseparability criteria [34], which is widely used in quantum optics as an entanglement witness. As shown in Fig. 4(c), we find that the first-steerability index $R^{(1)}$ is always greater than one, indicating a lack of steerability for all interaction time in the simulation.

Having found no linear entanglement, we proceed to investigate the nonlinear correlations. In Fig. 5, we plot the relation between the linear quadratures of mode $A$ and the expectation values of quadratic quadratures of mode $B$. From Fig. 5(a), we see a nonzero linear correlation between the quadratures $\hat{X}_{A}$ and $\hat{X}_{B}^{(2)}$ [also between $\hat{Y}_{A}$ and $\hat{Y}_{B}^{(2)}$ in Fig. 5(d)]. The linear correlations between cross quadratures $\left\langle\hat{X}_{A} \hat{Y}_{B}^{(2)}\right\rangle$ and $\left\langle\hat{Y}_{A} \hat{X}_{B}^{(2)}\right\rangle$ are zero, as seen in Figs. 5(b) and 5(c). From Fig. 5(a), we see that by measuring $\hat{X}_{A}$, we learn about the distribution of $X_{B}^{(2)}$. For the averaged distribution of $X_{B}^{(2)}$ one obtains a variance smaller than the "shot noise" variance given by the Heisenberg uncertainty principle. This is also true for the $\hat{Y}$ quadrature, which implies that the quadratic quadratures of mode $B$ is steerable by $A$. We plot the second-steerability index $R^{(2)}$ for different values of pump strength $\alpha_{c}$ against interaction time in Fig. 4(d). The results confirm that provided a shortenough interaction time, mode $B$ is steerable for all values of pump strength.

We make an estimate on the realizability of the nonlinear entanglement in a $\chi^{(3)}$ process enhanced by an optical cavity. In order for a sufficiently strong interaction between the modes in a cavity, we need an input pump power of the order $P_{\star}=P_{0}^{2} /\left(\hbar \omega_{C} \gamma\right)$, where $\gamma$ is the cavity decay rate and $P_{0}$ is the cavity threshold power parameter, which depends on the nonlinearity of the $\chi^{(3)}$ crystal, the cavity geometry, and its losses [16]. For a silicon nitride crystal with an observed third-order nonlinearity of $\chi^{(3)}=$ $10^{-20} \mathrm{~m}^{2} \mathrm{~V}^{-2}$ [35] at a pump frequency of $\omega_{C}=5.3 \times$ $10^{15} \mathrm{~s}^{-1}$ in a microcavity configuration of length $L=1 \mathrm{~mm}$, the cavity threshold power $P_{0} \sim\left(10^{3} \mathrm{~W}\right) T^{2}$, where $T=L \gamma / c$ parameterizes the losses in the cavity. Note that modes $A$ and $B$ are chosen to be nearly degenerate; hence, dispersion in the value of $\chi^{(3)}$ can be neglected. This puts the required input pump power at $P_{\star} \sim\left(6 \times 10^{12} \mathrm{~W}\right) T^{3}$. For a cavity with a finesse of $2 \times 10^{4}$, the required pump power for observation of nonlinear entanglement is of the order $P_{\star} \sim 1 \mathrm{~W}$, which is experimentally attainable. Considering that the propagation loss of silicon nitride can be as low as $0.05 \mathrm{~dB} / \mathrm{m}$ [36,37], a resonator with a round-trip length of $1 \mathrm{~mm}$ can theoretically achieve a finesse of over $5 \times 10^{5}$. Here we have assumed a relatively large mode diameter on the order of $10 \mu \mathrm{m}$. The power density in the resonator is thus comparable to what has been achieved experimentally [38].

In conclusion, we have introduced a method to quantify the entanglement between higher-order quadratures of a two-mode system, and have examined the process of threephoton down-conversion as a source of nonlinear entanglement. We showed that in this process, the quadraticlike correlations can be utilized to generate continuously tunable cat states. In certain regimes, these cat states can also be steered. This process is experimentally feasible and can be realized with a third-order nonlinear material in a high-finesse cavity. While we have presented a specific example for nonlinear entanglement, we anticipate that nonlinear quantum correlations will find many more applications in quantum information protocols.

This research is supported by the Australian Research Council (Projects No. CE110001027 and No. DP1092891).

*ping.lam@anu.edu.au

[1] A. Einstein, B. Podolsky, and N. Rosen, Phys. Rev. 47, 777 (1935).

[2] E. Schrödinger, Proc. Cambridge Philos. Soc. 31, 555 (1935).

[3] M. D. Reid, P. D. Drummond, W. P. Bowen, E. G. Cavalcanti, P. K. Lam, H. A. Bachor, U. L. Andersen, and G. Leuchs, Rev. Mod. Phys. 81, 1727 (2009).

[4] S. J. Jones, H. M. Wiseman, and A. C. Doherty, Phys. Rev. A 76, 052116 (2007).

[5] E. G. Cavalcanti, S. J. Jones, H. M. Wiseman, and M. D. Reid, Phys. Rev. A 80, 032112 (2009).

[6] A. Aspect, P. Grangier, and G. Roger, Phys. Rev. Lett. 49, 91 (1982). 
[7] A. Aspect, J. Dalibard, and G. Roger, Phys. Rev. Lett. 49, 1804 (1982).

[8] H. M. Wiseman, S. J. Jones, and A. C. Doherty, Phys. Rev. Lett. 98, 140402 (2007).

[9] D. J. Saunders, S. J. Jones, H. M. Wiseman, and G. J. Pryde, Nat. Phys. 6, 845 (2010).

[10] C. M. Caves and B. L. Schumaker, Phys. Rev. A 31, 3068 (1985).

[11] M. D. Reid and P. D. Drummond, Phys. Rev. Lett. 60, 2731 (1988).

[12] C. Silberhorn, P. K. Lam, O. Weiss, F. König, N. Korolkova, and G. Leuchs, Phys. Rev. Lett. 86, 4267 (2001).

[13] J. Laurat, T. Coudreau, G. Keller, N. Treps, and C. Fabre, Phys. Rev. A 71, 022313 (2005).

[14] D. H. Smith et al., Nat. Commun. 3, 625 (2012).

[15] V. Händchen, T. Eberle, S. Steinlechner, A. Samblowski, T. Franz, R. F. Werner, and R. Schnabel, Nat. Photonics 6, 598 (2012).

[16] T. Felbinger, S. Schiller, and J. Mlynek, Phys. Rev. Lett. 80, 492 (1998).

[17] S. Boixo, S. T. Flammia, C. M. Caves, and J. M. Geremia, Phys. Rev. Lett. 98, 090401 (2007).

[18] S. Boixo, A. Datta, S. T. Flammia, A. Shaji, E. Bagan, and C. M. Caves, Phys. Rev. A 77, 012317 (2008).

[19] A. Ourjoumtsev, F. Ferreyrol, R. Tualle-Brouri, and P. Grangier, Nat. Phys. 5, 189 (2009).

[20] N. Sangouard, C. Simon, N. Gisin, J. Laurat, R. TualleBrouri, and P. Grangier, J. Opt. Soc. Am. B 27, A137 (2010).

[21] J. S. Neergaard-Nielsen, Y. Eto, C. W. Lee, H. Jeong, and M. Sasaki, Nat. Photonics 7, 439 (2013).

[22] S. Song, C. M. Caves, and B. Yurke, Phys. Rev. A 41, 5261 (1990).
[23] A. Ourjoumtsev, R. Tualle-Brouri, J. Laurat, and P. Grangier, Science 312, 83 (2006).

[24] A. Ourjoumtsev, H. Jeong, R. Tualle-Brouri, and P. Grangier, Nature (London) 448, 784 (2007).

[25] A. I. Lvovsky, R. Ghobadi, A. Chandra, A. S. Prasad, and C. Simon, Nat. Phys. 9, 541 (2013).

[26] See Supplemental Material at http://link.aps.org/ supplemental/10.1103/PhysRevLett.114.100403, which includes Refs. [1,3-5,8,27,28,33,34], for more details of the generation of the cat state and the link to an EPR paradox.

[27] R. Simon, Phys. Rev. Lett. 84, 2726 (2000).

[28] E. G. Cavalcanti and M. D. Reid, Phys. Rev. Lett. 97, 170405 (2006); , 77, 062108 (2008).

[29] M. B. Plenio and S. Virmani, Quantum Inf. Comput. 7, 001 (2007).

[30] V. Bužek and G. Drobný, Phys. Rev. A 47, 1237 (1993).

[31] M. Koniorczyk, J. Janszky, and Z. Kis, Acta Phys. Slovaca 49, 707 (1999).

[32] M. Hillery, Phys. Rev. A 36, 3796 (1987).

[33] M. D. Reid, Phys. Rev. A 40, 913 (1989).

[34] Lu-Ming Duan, G. Giedke, J. I. Cirac, and P. Zoller, Phys. Rev. Lett. 84, 2722 (2000).

[35] T. Ning, O. Hyvärinen, H. Pietarinen, T. Kaplas, M. Kauranen, and G. Genty, Opt. Express 21, 2012 (2013).

[36] J. F. Bauters, M. J. R. Heck, D. D. John, J. S. Barton, C. M. Bruinink, A. Leinse, R. G. Heideman, D. J. Blumenthal, and J. E. Bowers, Opt. Express 19, 24090 (2011).

[37] D. T. Spencer, J. F. Bauters, M. J. R. Heck, and J. E. Bowers, Optica 1, 153 (2014).

[38] M. Eichenfield, C. P. Michael, R. Perahia, and O. Painter, Nat. Photonics 1, 416 (2007). 